\title{
SHAPES OF STARS
}

\author{
SADAYOSHI KOJIMA AND YASUSHI YAMASHITA
}

(Communicated by Jonathan M. Rosenberg)

\begin{abstract}
In this paper we construct a natural geometric structure for the space of shapes of a star-shaped polygon. Roughly speaking we find: The set of similarity classes of marked stars forms a hyperbolic right angle pentagon bundle over the space of external angle sets of inscribed pentagons. The assignment of the shape of its fiber to each angle set forms a hyperbolic plane bundle over the Teichmüller space of hyperbolic right angle pentagons. Any automorphism induced by renumbering is compatible with these geometric structures.
\end{abstract}

\section{INTRODUCTION}

This work is motivated by Thurston's ingenious description of the set of Euclidean structures on the 2-sphere with prescribed cone singularities in [2]. It was shown to admit a complex hyperbolic cone manifold structure very naturally. The result leads us to the thesis that the set of shapes of an appropriate space should admit naturally a geometric structure. We present here by a very elementary argument one such example based on the real cut version of Thurston's description.

The object is a star as in the title, namely, a star-shaped polygon spanned by five vertices on the plane. It is identified with the configuration of five points in the plane that span a convex pentagon. To state our natural geometrization on the set of such shapes, it is necessary to clarify the notion, but roughly speaking we show:

Theorem. The set of similarity classes of marked stars forms a hyperbolic right angle pentagon bundle over the space of external angle sets of inscribed pentagons. The assignment of the shape of its fiber to each angle set forms a hyperbolic plane bundle over the Teichmüller space of hyperbolic right angle pentagons. Any automorphism induced by renumbering is compatible with these geometric structures.

\section{Proof}

Let $\mathscr{S}$ be the space of marked stars up to similarity transformations, with the natural topology. This is the set of shapes of marked stars that we want to

Received by the editors April 5, 1991 and, in revised form, June 17, 1991.

1980 Mathematics Subject Classification (1985 Revision). Primary 57M50; Secondary 53C15.

Key words and phrases. Similarity class, geometric structure, hyperbolic geometry, moduli space. 


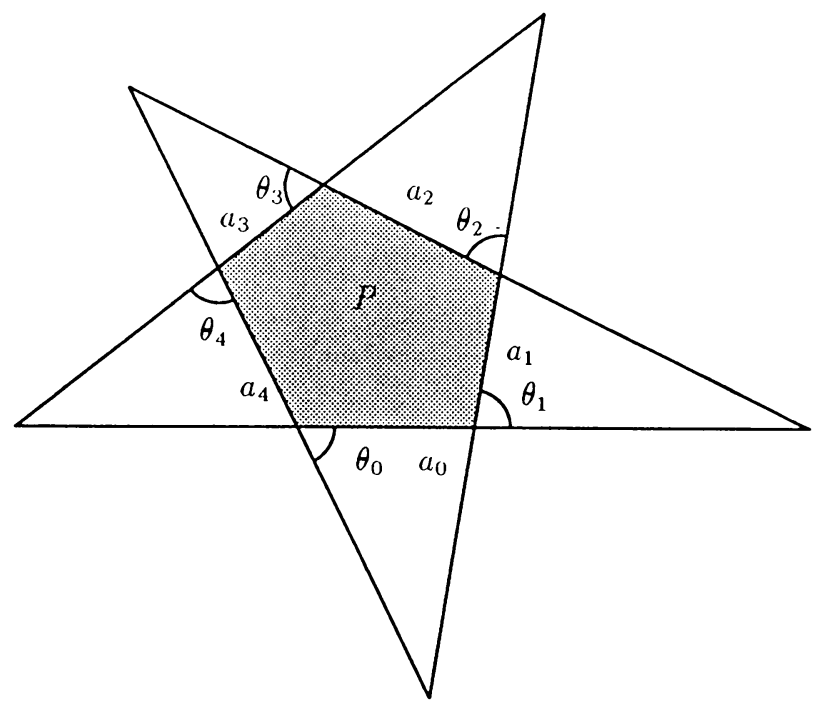

FIGURE 1

geometrize. It is obvious that there is six-dimensional freedom for perturbations. Remarking the vertices induces an automorphism of $\mathscr{S}$. Those automorphisms form a group $G=\operatorname{Aut}(\mathscr{S})$, which is isomorphic to the dihedral group of order 10.

The inscribed polygon $P$ spanned by the intersections of edges is also a convex pentagon. The shape of a star corresponds to the shape of $P$ bijectively, and hence the space we will geometrize is identified with the set of similarity classes of $P$ 's. We prefer to work with $P$ rather than with a star itself.

Define the edges of $P$ by $a_{0}, a_{1}, \ldots, a_{4}$ and corresponding external angles by $\theta_{0}, \theta_{1}, \ldots, \theta_{4}$ in counterclockwise order (see Figure 1). Then the angles must satisfy the relations $\theta_{j}>0$ for all $j, \sum_{j} \theta_{j}=2 \pi$ and $\theta_{j}+\theta_{j+1}<\pi$ for all $j$. The indices should be understood modulo 5 throughout the sequel. We denote the space of sets of possible external angles by $\Theta=\left\{\left(\theta_{0}, \ldots, \theta_{4}\right)\right\}$. It is homeomorphic to an open 4-cell.

Choose $\theta=\left(\theta_{0}, \ldots, \theta_{4}\right) \in \boldsymbol{\Theta}$. We use the notation $a_{j}$ to indicate the length of $a_{j}$ also. Then they are also subject to the equation $\sum_{j=0}^{4} a_{j} \exp \left(i \sum_{k=0}^{j} \theta_{k}\right)$ $=0$, where $i=\sqrt{-1}$. Taking its real and imaginary parts, we get two linear equations over the real numbers. Let $V_{\theta}$ be the subspace of a five-dimensional Euclidean space $\mathbf{V}=\left\{\left(a_{0}, \ldots, a_{4}\right)\right\}$ defined by these equations. By the angle condition, it will be a three-dimensional space. Its intersection with the first orthant $\mathbf{V}^{+}=\left\{a_{j}>0\right.$ for all $\left.j\right\}$ is the space of inscribed pentagons of stars with angle $\theta=\left(\theta_{0}, \ldots, \theta_{4}\right)$.

The set of similarity classes of $P$ 's with a prescribed angle set $\theta$ is then the quotient of $\mathbf{V}^{+} \cap V_{\theta}$ by dilation. Thurston identified this quotient with a level surface of the area function in [2]. Here we recall his argument for this setting. Choose a triangle $A_{j}$ in the star spanned by edges $a_{j}, a_{j+2}, a_{j+3} . \overline{A_{j}-P}$ then consists of the right-hand triangle $B_{j}$ and the left-hand triangle $C_{j}$. See Figure 2.

Fixing $\theta$, we regard areas of these triangles as functions of $a_{j}$ 's. Let us 


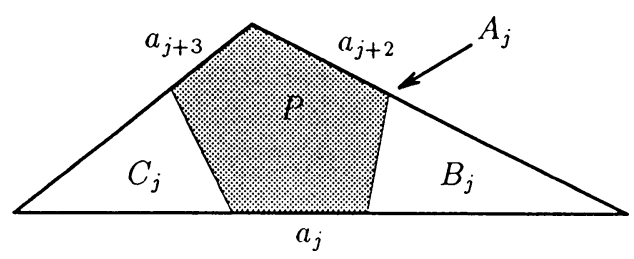

FIGURE 2

denote the square roots of the areas of $A_{j}, B_{j}$, and $C_{j}$ by $X_{j}, Y_{j}$, and $Z_{j}$ respectively. $Y_{j}$ and $Z_{j}$ are some constant multiples of $a_{j+1}$ and $a_{j+4}$, while $X_{j}$ is a linear combination of $a_{0}, \ldots, a_{4}$. We obviously have the formula $\operatorname{Area}_{\theta}(P)=X_{j}^{2}-Y_{j}^{2}-Z_{j}^{2}$, where the value does not depend on $j$. $\operatorname{Area}_{\theta}=$ $X_{j}^{2}-Y_{j}^{2}-Z_{j}^{2}$ is then regarded as a quadratic form on $V_{\theta}$ with signature (1, 2). The level surface $\operatorname{Area}_{\theta}^{-1}(1)$ is a hyperboloid in $V_{\theta}$, which has a hyperbolic structure with respect to the metric - Area $_{\theta}$. Then the real cut version of Thurston's description, of which we give a self-contained proof, is as follows.

Lemma 1. $\mathbf{V}^{+} \cap \operatorname{Area}_{\theta}^{-1}(1) \subset V_{\theta}$ is the interior of a right angle geodesic pentagon. Proof. $\mathbf{V}^{+} \cap \operatorname{Area}_{\theta}^{-1}(1)$ is in fact the domain in $\operatorname{Area}_{\theta}^{-1}(1) \subset V_{\theta}$ bounded by the $\left\{a_{j}=0\right\}$ 's. Since each $\left\{a_{j}=0\right\}$ is a hypersurface in $V_{\theta}$, its intersection with $\operatorname{Area}_{\theta}^{-1}(1)$ is a geodesic. Identify the projective model disk with the unit disk in $X_{j}=1$ with respect to the coordinates $\left(X_{j}, Y_{j}, Z_{j}\right)$ on $V_{\theta}$ and project this domain there. $\left\{a_{j+1}=0\right\}$ projects on the $Z_{j}$-axis and $\left\{a_{j+4}=0\right\}$ on the $Y_{j}$-axis. They intersect at the origin, which shows that these two geodesics make a right angle. Since we had no preference for the choice of $j$, this proves the claim by shuffling the marking.

Each inverse image of the projection $\pi: \mathscr{S} \rightarrow \Theta$ is thus the interior of a hyperbolic pentagon in $\operatorname{Area}^{-1}(1) \subset V_{\theta} \subset \mathbf{V}$. The natural topology on $\mathscr{S}$ is identified with the relative topology on $\mathscr{S}$ in the product of $\Theta$ with the canonical bundle over the Grassmann manifold of three-dimensional subspaces in $\mathbf{V}$, and the projection $\pi: \mathscr{S} \rightarrow \Theta$ becomes a fibration.

Now, assigning the shape of $\pi^{-1}(\theta)$ to each $\theta$, we get the map $\eta$ of $\Theta$ to the space of marked hyperbolic right angle pentagons. This map is what we want to geometrize next. Nothing is obvious a priori, and we start by describing the target space.

Let $\mathscr{T}$ be the space of marked hyperbolic right angle pentagons, which is a socalled Teichmüller space. Since a right angle pentagon is determined by two edge lengths, it is a two-dimensional space and is in fact homeomorphic to a 2-cell, as we will see soon. $\mathscr{T}$ can be identified with the space of discrete representations of a Coxeter group $\left\langle r_{0}, \ldots, r_{4}\right| r_{j}^{2}=\left(r_{j} r_{j+1}\right)^{2}=1$ for all $\left.j\right\rangle$ to $\operatorname{PSL}(2, \mathbf{R})$ up to conjugacy. $\mathscr{T}$ has a natural dihedral symmetry by renumbering.

Here is another description of $\mathscr{T}$ that does not respect the dihedral symmetry. Put a marked hyperbolic pentagon in the projective model located on the unit disk so that a preferred vertex equals the origin and two adjacent edges are on the axes as in Figure 3 on the next page. Let $(p, 0)$ and $(0, q)$ be the Euclidean coordinates of the intersections between the axes and the other adjacent edges. These are subject to the relation $p^{2}+q^{2}>1$ to give rise to 


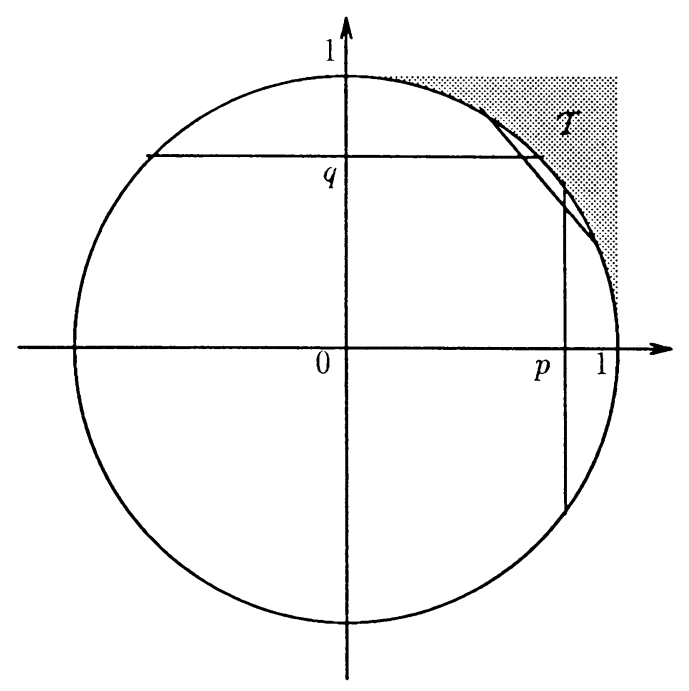

FIGURE 3

a hyperbolic right angle pentagon. Conversely, we can construct a unique hyperbolic pentagon whenever this inequality holds. Hence $\mathscr{T}$ can be identified with the point set $\left\{(p, q) ; 0<p, q<1\right.$ and $\left.p^{2}+q^{2}>1\right\}$ in the plane.

To figure out the shape of $\pi^{-1}(\theta)$, we put it in the projective disk model on $X_{j}=1$ in $V_{\theta}$ as in the proof of Lemma 1 and find the coordinates of the other intersections. We first let $p_{j}$ be the $Z_{j}$-coordinate of $\left\{a_{j+1}=0\right\} \cap\left\{a_{j+3}=0\right\}$ in the disk and $q_{j}$ the $Y_{j}$-coordinate of $\left\{a_{j+4}=0\right\} \cap\left\{a_{j+2}=0\right\}$. To compute these intersections, think of the external moments $a_{j+1}=a_{j+3}=0$ and $a_{j+4}=$ $a_{j+2}=0$. They correspond to the degenerate $P$ 's.

At the first moment, $B_{j}$ degenerates and $A_{j}$ consists of $P$ and $C_{j}^{e}$, while at the last moment $C_{j}$ degenerates and $A_{j}$ consists of $P$ and $B_{j}^{e}$ like in Figure 4. Then $\sqrt{\text { Area } C_{j}^{e}}$ is the $Z_{j}$-coordinate of the intersection $\left\{a_{j+1}=\right.$ $0\} \cap\left\{a_{j+3}=0\right\}$ in $\operatorname{Area}_{\theta}^{-1}(1) \subset V_{\theta}$ and $\sqrt{\text { Area } B_{j}^{e}}$ is the $Y_{j}$-coordinate of $\left\{a_{j+4}=0\right\} \cap\left\{a_{j+2}=0\right\}$. Hence projecting these points to the unit disk in $X_{j}=1$ and recalling the normalization Area $P=1$, we get

$$
p_{j}^{2}=\frac{\text { Area } C_{j}^{e}}{\text { Area } C_{j}^{e}+1}=\frac{\text { base width of } C_{j}^{e}}{\text { base width of } A_{j}}
$$

and

$$
q_{j}^{2}=\frac{\text { Area } B_{j}^{e}}{\text { Area } B_{j}^{e}+1}=\frac{\text { base width of } B_{j}^{e}}{\text { base width of } A_{j}}
$$

These identities give us a method of computing $p_{j}$ and $q_{j}$ just by looking at a picture (Figure 5); namely, given $P$, take the unit interval $I=[0,1]$ on the real axis of the complex plane $\mathbf{C}$, and draw the similar triangles to $A_{j} \supset B_{j}^{e}, C_{j}^{e}$ based on $I$. Then it turns out that the coordinate of the vertex of $C_{j}^{e}$ on $(0,1)$ is $p_{j}^{2}$ and that of $B_{j}^{e}$ is $1-q_{j}^{2}$. Using this method we prove 

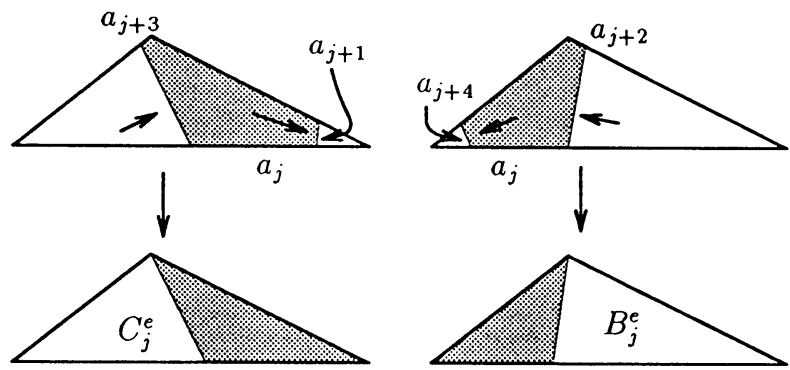

FIGURE 4

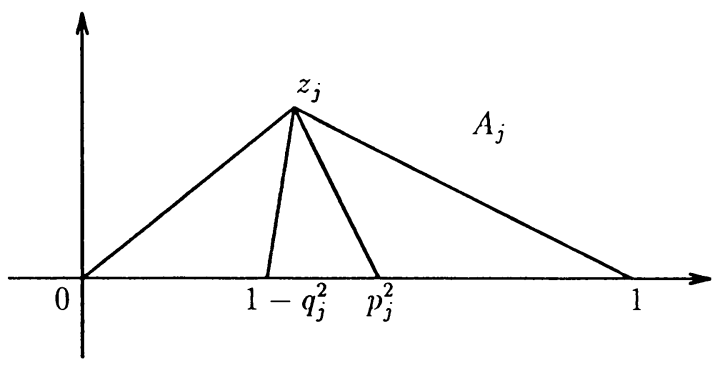

FIGURE 5

Lemma 2. Choose $0<p, q<1$ satisfying $p^{2}+q^{2}>1$. Then the space of sets of angles $\theta$ so that $p_{j}=p$ and $q_{j}=q$ can be identified with a hyperbolic plane. Proof. Given $p$ and $q$, plot $0<1-q^{2}<p^{2}<1$ on $I$ and any $z_{j}$ in the upper halfspace of $C$. Joining four points on $I$ with $z_{j}$, we get triangles that are identical with some $A_{j} \supset B_{j}^{e}, C_{j}^{e}$. From this triangle, we can read off those $\theta$ that have the property that $p_{j}=p$ and $q_{j}=q$.

The freedom for $\theta=\left(\theta_{0}, \ldots, \theta_{4}\right)$ to have $p_{j}=p$ and $q_{j}=q$ is measured by the position of $z_{j}$ in the upper halfspace. The set of such angles is hence identified with the upper halfspace, a model of the hyperbolic plane.

The hyperbolic structure on the fiber by this identification in fact depends on $j$ a priori, but it turns out to be independent of the choice of $j$ because another complex coordinate is transformed by linear fractional transformations generated by

$$
z_{j+1}=\frac{\left(1-q_{j}^{2}\right)\left(z_{j}-p_{j}^{2}\right)}{p_{j}^{2}\left(z_{j}-1+q_{j}^{2}\right)} .
$$

This can be seen simply by rotating and rescaling the shaded triangle similar to $A_{j+1}$ in Figure 6 on the next page, so that the base edge becomes $[0,1]$ in C.

In particular, the map $\eta: \Theta \rightarrow \mathscr{T}$ becomes a hyperbolic plane fibration. Recall that the automorphism group $G=\operatorname{Aut}(\mathscr{S})$ is generated by the maps corresponding to a cyclic renumbering $\sigma=(01234)$ and a reflective renumbering $\tau=(01)(24)(3)$. The action of $G$ descends to an effective action by shuffling suffixes on $\Theta$. The action of $G$ on $\Theta$ commutes with the fibration $\eta: \boldsymbol{\theta} \rightarrow \mathscr{T}$. 


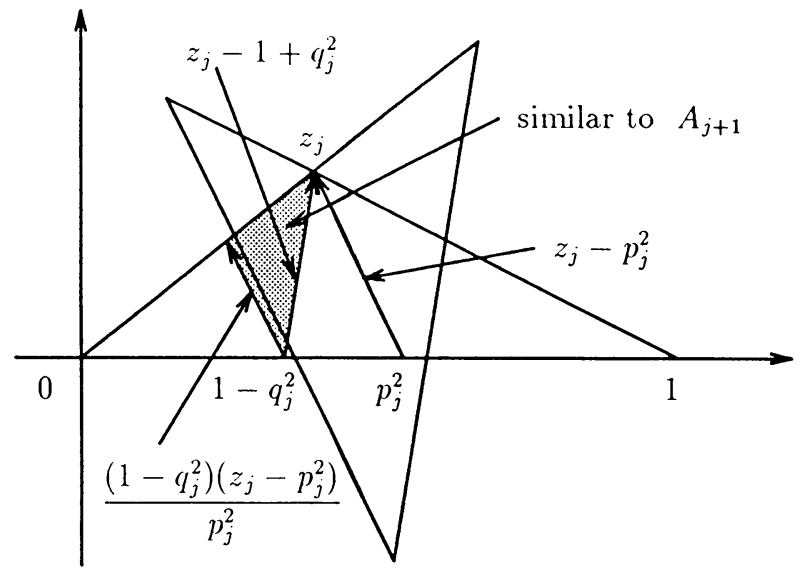

FIGURE 6

Lemma 3. $G=\operatorname{Aut}(\mathscr{S})$ acts on each fiber as an isometry with respect to its hyperbolic structure.

Proof. Choose uniform $j$ to identify a fiber with the upper halfspace, and let $z$ and $w$ be the complex coordinates on the fibers of $\alpha \in \mathscr{T}$ and $\sigma_{*}(\alpha)$ or $\tau_{*}(\alpha)$. Then the induced automorphisms of $\sigma$ and $\tau$ on the fiber are linear fractional transformations,

$$
\sigma_{*}(z)=\frac{\left(1-q_{j}^{2}\right)\left(w-p_{j}^{2}\right)}{p_{j}^{2}\left(w-1+q_{j}^{2}\right)} \quad \text { and } \quad \tau_{*}(z)=-\bar{w}+1,
$$

by the same calculation as before. Hence they are isometries with respect to the hyperbolic metric we gave.

This together with Lemmas 1 and 2 completes the proof of Theorem.

The quotient space $\mathscr{S} / G$ is now described as a hyperbolic pentagon orbifold bundle over the moduli $\Theta / G$ of angle sets. $\Theta / G$ itself is regarded as a hyperbolic plane bundle over $\mathscr{T} / G$. As a final remark, we relate $\Theta / G$ with the figure eight knot, which quite often appears in the study of hyperbolic manifolds.

Consider the embedding $l: \mathscr{T} \rightarrow \mathbf{R}^{5}$ by assigning the $j$ th coordinate to the length of an edge on $\left\{a_{j}=0\right\}$. The action of $G=\operatorname{Aut}(\mathscr{S})$ on $\mathbf{R}^{5}$ by shuffling coordinates is compatible with the dihedral action on $l(\mathscr{T})$. The equilateral hyperbolic right angle pentagon $\alpha_{0}$ represents the unique fixed point of the action. Since $G$ fixes $\alpha_{0}$, the tangent space of $l(\mathscr{T})$ at $\alpha_{0}$ is a $G$-invariant two-dimensional subspace in $\mathbf{R}^{5}$. By linear algebra, we can find two $G$-invariant planes in $\mathbf{R}^{5}$ spanned by the orbit of the real part of $\left(1, \zeta, \zeta^{2}, \zeta^{3}, \zeta^{4}\right)$ or that of $\left(1, \zeta^{2}, \zeta^{4}, \zeta, \zeta^{3}\right)$, where $\zeta=\exp (2 \pi i / 5) . \sigma_{*}$ acts on the first plane as a rotation of $2 \pi / 5$ and the second by $4 \pi / 5$. By perturbing the equilateral pentagon slightly, we can conclude that the tangent space of $l(\mathscr{T})$ at $\alpha_{0}$ is the first one. Hence the tangential representation of the $G$-action on $\mathscr{T}$ at $\alpha_{0}$ is identified with the dihedral action on the complex plane $\mathbf{C}$ so that $\sigma_{*}$ is the multiplication by $\zeta$ and $\tau_{*}$ is the complex conjugation.

Since $\alpha_{0}$ is a fixed point, its fiber $\eta^{-1}\left(\alpha_{0}\right)$ is invariant by $G$. The equiangular angle set $\beta$ represents the unique fixed point. The tangential representation 
of the $G$-action on $\eta^{-1}\left(\alpha_{0}\right)$ at $\beta$ is identified with the dihedral action on $\mathbf{C}$ so that $\sigma_{*}$ is the multiplication by $\zeta^{3}$, by the computation in Lemma 3.

Hence the $G$-action on $\Theta$ at $\beta$ is tangentially described by maps on $\mathbf{C}^{2}$,

$$
\sigma_{*}(x, y)=\left(e^{2 \pi i / 5} x, e^{-4 \pi i / 5} y\right) \text { and } \tau_{*}(x, y)=(\bar{x}, \bar{y}) .
$$

The $G$-action on $\Theta$ itself is not a product with respect to our trivialization; however, it is fiber-preserving and hence equivariant to the tangential representation.

The action on $\mathbf{C}^{2}$ induces the isometric action on the unit sphere with respect to the spherical metric. The quotient by $\left\langle\sigma_{*}\right\rangle$ is the lens space $L(5,2) \cong$ $L(5,3)$. $\tau_{*}$ descends to an isometric involution leaving a closed geodesic fixed. The further quotient by $\tau_{*}$ becomes a spherical orbifold with a knot as a branched singularity of order 2. More detailed study, for instance in [1], shows that the underlying space is homeomorphic to the 3-sphere and the singular locus is the figure eight knot. Now, since the action on $\mathbf{C}^{2}$, which is equivariant to the $G$-action on $\Theta$, is just an open cone extension of the action on the unit sphere, we have proved

Lemma 4. $\boldsymbol{\theta} / G$ is isomorphic as an orbifold to the open cone of the spherical orbifold on the 3-sphere with the figure eight knot as a branched singularity of order 2 .

\section{REFERENCES}

1. M. Sakuma, The geometries of spherical Montesinos links, Kobe J. Math. 7 (1990), 167-190.

2. W. P. Thurston, Shapes of polyhedra, preprint, 1987.

Department of Information Science, Tokyo Institute of Technology, Ohokayama, MEgURO, TOKYO 152, JAPAN

E-mail address: sadayosi@is.titech.ac.jp

Department of INformation ScIence, Nara Women's University, Kita-Uoya Nishimachi, NARA 630, JAPAN

E-mail address: yamasita@is.titech.ac.jp 